\title{
Empowering Critical Thinking Skills With Computerized Patient Simulators
}

Francisca Cisneros Farrar, Austin Peay State University, USA

Leslie Suggs, Austin Peay State University, USA

\begin{abstract}
Students struggle with the mastery of critical thinking skills which are essential to their academic success. University faculty are challenged to create teaching strategies to help students build critical thinking skills. Nursing faculty at Austin Peay State University in Clarksville, Tennessee looked to research and technology for ways to empower critical thinking skills in students. Faculty found computerized patient simulators were a best practice methodology that empowers mastery of critical thinking skills. This evidence-based integrative teaching format allows the process of analyzing, synthesizing, evaluation, and applying information gathered through observation, experience, communication, and reflection. This simulated interactive format also allows realistic training from basic skills to advanced life-saving skills. The Institute of Medicine recommends the use of simulators to create a culture of safety. Simulation creates a culture of safety for the learner because the realistic training scenario is without risk to patients and healthcare providers. A simple to complex scenario comes alive with simulation. The learners in the scenario are videotaped. Afterward debriefing is done with reflective thinking. The simulation experience is a fun and highly motivated experience conducted in a nonthreatening environment. The learner is able to analyze the experience and learn to improve their skills. Austin Peay State University nursing faculty identified four simulators that can help learners master critical thinking skills. These simulators are SimMan, SimBaby, Novelle Maternal and Neonatal Birthing Simulator, and Comat Sim. These simulators can be used in a variety of clinical settings. Simulators can also be used to teach critical thinking skills in sociology, psychology, and homeland security.
\end{abstract}

Keywords: SimMan, patient simulator, nursing, critical thinking skills

\section{SIMMAN}<smiles>[C]1C=CCCC1</smiles>

imMan is a computerized patient simulator that has realistic anatomy and clinical functionality of an adult male. Computerized case scenarios provide a realistic interactive teaching format. The learner is challenged to master competency skills during the scenario. If the learner does not intervene correctly, the simulator develops complications including death. SimMan can be used to master basic fundamental skills to advanced life support skills in a critical care course. (Laerdal 2010) Mental health and communication skills can also be simulated. For example, a SimMan scenario can be programmed to manifest symptomatology of paranoia or suicidal ideation. SimMan can be dressed to reflect a clinical presentation of a patient with a psychiatric disorder. Community health skills can also be mastered by developing a scenario involving symptomatology of small pox or poison. Simulation experiences can be developed for any type of experience. The SimMan interactive format allows the practice of teamwork, leadership, delegation, prioritization, and communication which are the building blocks of critical thinking skills.

\section{SIMBABY}

SimBaby is a portable advanced infant simulator for team training. It has realistic anatomy and clinical functionality for a baby that enables simulation training. SimBaby has interactive technology that allows learners to 
practice basic routine newborn care to pediatric emergency care scenarios. Mastery of infant skills includes neonatal infection, prematurity, and respiratory distress. (Laerdal 2010)

\section{NOVELLE MATERNAL AND NEONATAL BIRTHING SIMULATOR}

This simulator is designed to provide the complete birthing experience before, during, and after delivery. Features include full size articulating female manikin with intubable airway with chest rise. The simulator has an intravenous arm for multiple meds and fluids, an automatic birthing system, an articulating birthing baby with placenta, and a resuscitation baby with intubable airway. (Guamard, 2010). The learner can build competency skills in an interactive realistic birthing process from a routine delivery with actual cervical dilation to complex maternal complications including forceps, assisted delivery, and C-section. (Gaumard, 2010). Case scenarios provide a realistic interactive no harm environment to the learner in their quest for critical thinking skills that build in complexity.

\section{COMBATSIM}

This simulator is a highly sophisticated simulator that mirrors the human body. This simulator is used by the military in training of emergency and trauma care of soldiers on the front line in a war zone. CombatSim is the "mother" of all simulators. This high fidelity patient simulator is designed for training in anesthesia, respiratory, and critical care. Highly developed features include pupils that dilate and restrict to light, response to administered drugs, automatic response to needle decompression of a tension pneumothorax, chest tube drainage, and automatic control of urine output. (Laerdal, 2010) Case scenarios for this high level training and mastery of advanced critical thinking skills include severe trauma disaster management, and bioterrorism response. The CombatSim safely builds advanced skills, and fosters collaboration with military and other disaster management personnel.

\section{EMPOWERMENT OF CRITICAL THINKING SKILLS}

Simulators offer valuable clinical experiences to learners while allowing a reliable and safe environment for the practice of basic skills to advanced life support skills. Simulations provide opportunities for rarely encountered skills also. Case scenarios can be developed for a variety of clinical settings including mental health, community, pharmacology, and disaster management. This interactive teaching strategy fosters collaboration and improved communication skills during and after the experience. The simulation process allows experiences in prioritization, delegation, time management, and problem solving. Debriefing of the case scenario interventions is analyzed with reflective thinking. This interactive simulation experience empowers critical thinking skills in a fun learning environment. Simulators are a valuable teaching strategy that facilitates learners' quest for mastery of critical thinking skills.

\section{PEDAGOGICAL EXAMPLE}

Research validates that poor communication can be a contributing factor in human error. To address this communication safety issue, mental health nursing faculty integrated simulation technology as a best practice methodology into the psychiatric mental health nursing curriculum. Faculty integrate four case scenarios using simulation into students' mental health clinical labs. The simulation experience focuses on mastery of 1) effective therapeutic communication techniques, 2) symptomatology of the four major mental health disorders, and 3) best practice interventions for the symptomatology using a holistic approach including physical, mental, spiritual, and environment care. The scenarios also link medical, safety, and psychiatric nursing issues.

At the end of the student's mental health clinical rotation, students are challenged to be creative, and asked to demonstrate mastery of clinical outcomes. In a small group simulation activity, students create a therapeutic communication script based on a conversation they had with an actual mental health patient. Based on emergent symptomatology from the communication script, students create and run a case scenario. A student leader coordinates the scenario and provides the voice for the simulator. Students run the case scenario in a fun and nonthreatening environment. Students are allowed to experiment with therapeutic communication skills, and apply 
best practice interventions that are not harmful to the mental health patient or the student. In the debriefing session, faculty are able to evaluate student mastery of skills, and provide constructive feedback for growth.

\section{REFERENCES}

1. Gaumard. (2010). Simulators for Health Care Education. Retrieved March 19, 2010 from http://www.gaumard.com

2. Laerdal. (2010). Laerdal SimMan Press Packet. Retrieved March 19, 2010 from http://www.laerdal.com 
NOTES 